\begin{abstract}
Nanocrystalline diamond films have generated substantial interest in recent years due to their low cost, extreme properties and wide application arena. Diamond is chemically inert, has a wide electrochemical window and is stable in numerous harsh environments. Nanocrystalline diamond has the advantage of being readily grown on a variety of substrates at very low thickness, resulting in smooth conformal coatings with high transparency. These films can be doped from highly insulating to metallically conductive and at very high concentrations become superconducting.
\end{abstract}

\title{
INTRODUCTION
}

Diamond has a number of properties that make it ideal for biological applications. The extreme chemical inertness, tuneable surface wettability, wide electrochemical window and high transparency are well documented properties ${ }^{1}$. More specific to diamond is the stability of functionalisation strategies, crucial for long term bio-sensing ${ }^{2}$. Hydrogen terminated diamond also exhibits $\mathrm{p}$ type surface conductivity ${ }^{3}$ and strong $\mathrm{pH}$ sensitivity ${ }^{4}$.

\section{EXPERIMENTAL PROCEDURE AND RESULTS}

Nanocrystalline diamond films were grown by Microwave Plasma Enhanced Chemical Vapour Deposion (MWPEVCD). Prior to deposition, substrates were immersed in ethanol / diamond powder suspensions and bath ultrasonicated for 30 mins. Growth parameters were 1-3\% $\mathrm{CH}_{4}$ in $\mathrm{H}_{2}$ gas phase, $2-3.5 \mathrm{~kW}, 20-40$ torr, $700{ }^{\circ} \mathrm{C}$ for a duration depending on desired thickness. Temperature was monitored by two colour pyrometry. Doping was achieved by the addition of trimethylboron (TMB) to the gas phase. Figure 1 shows a plot of growth rate against substrate temperature at $700{ }^{\circ} \mathrm{C}$ and $3500 \mathrm{~W}$. It can be seen from this figure that the growth rate increases with $\mathrm{CH}_{4}$ fraction as expected. 


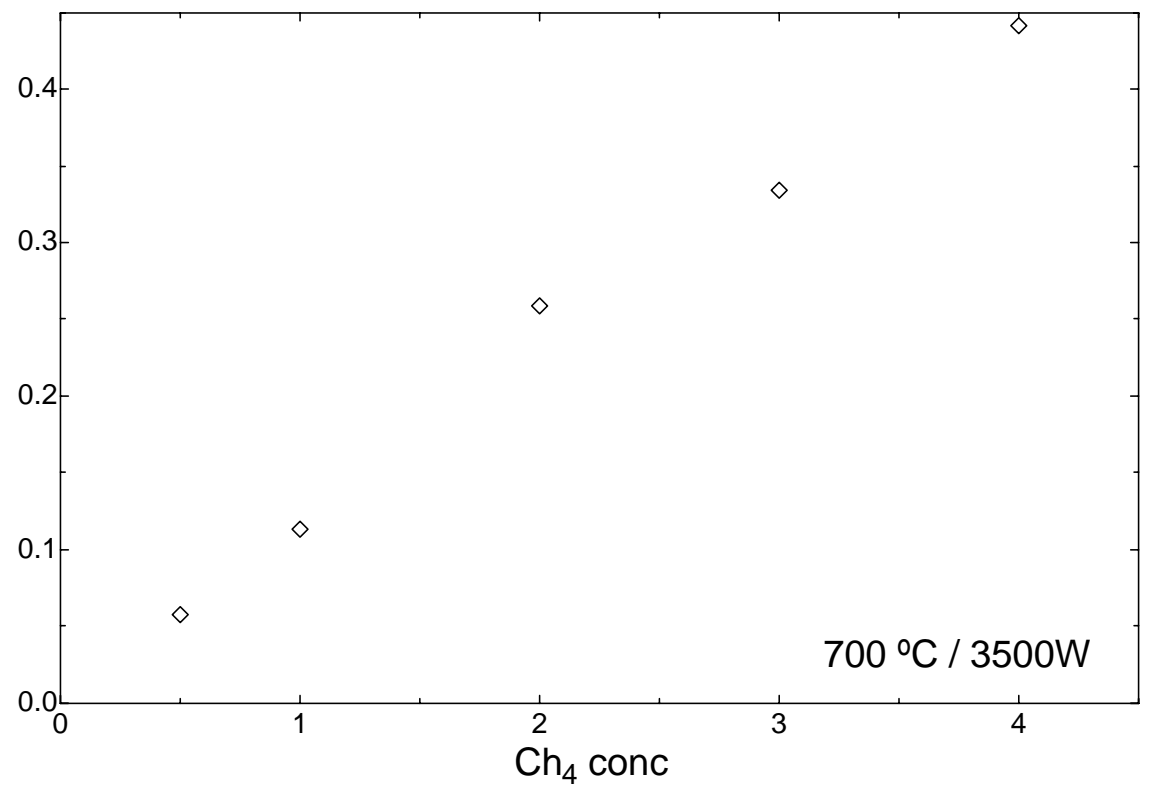

Figure 1: Growth rate against methane fraction for nanocrystalline diamond deposition at $700{ }^{\circ} \mathrm{C}$ and $3500 \mathrm{~W}$.

Figure 2 (a) is a Scanning Electron Microscopy image of a standard film of NCD grown from $1 \% \mathrm{CH}_{4}$ in $\mathrm{H}_{2}$, this film exhibits one colour uniformity over 2 ". This film exhibits grain sizes less than 50nm and is around 50nm thick. The grain size is reasonably uniform in this film, and it is found that films grown with higher methane content usually show a much wider distribution of crystallite size.
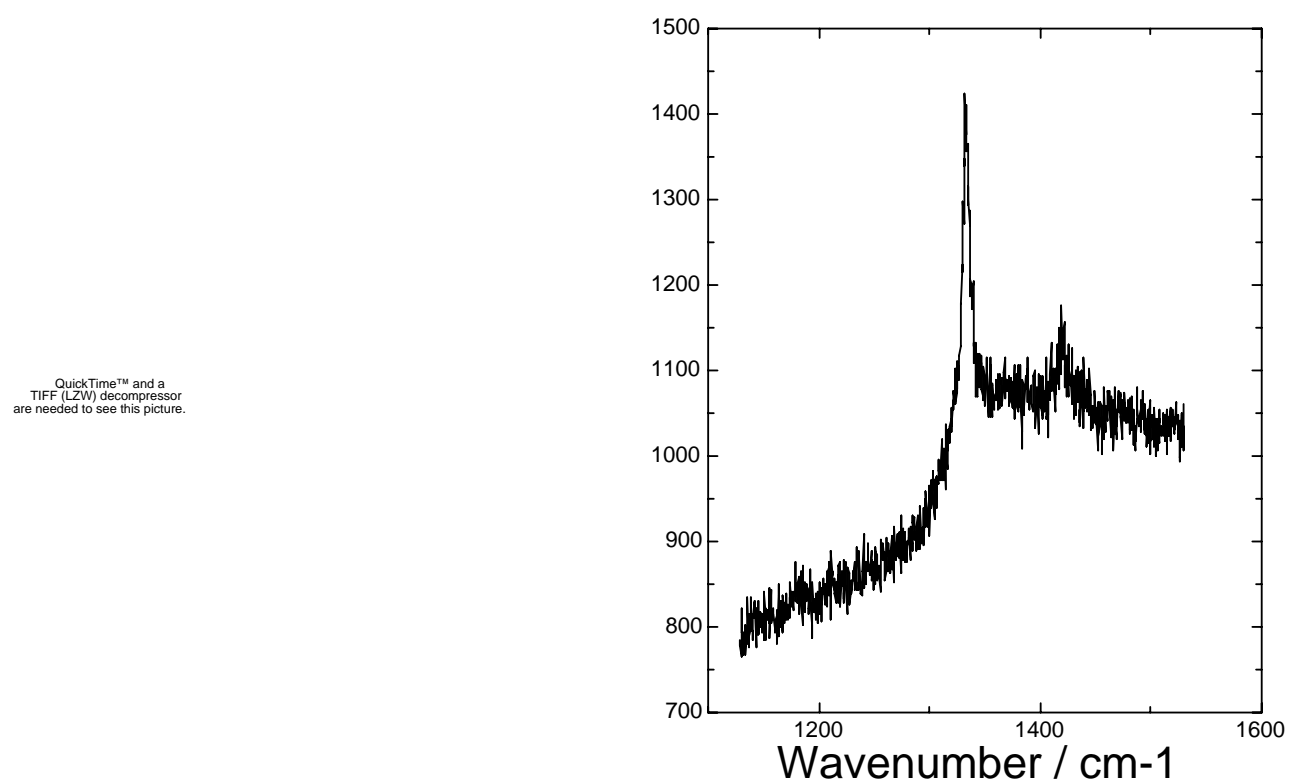

Figure 2: (a) Scanning Electron Micrograph and (b) Raman spectrum of nanocrystalline diamond film 
This NCD obviously shows clear faceting at this length scale unlike UNCD [ref]. Figure 2(b) is a Raman spectrum of a general NCD filom. It can be seen that unlike UNCD, NCD exhibits clear $\mathrm{sp}^{3}$ bonding ion Raman due to its strong crystalline faceting as seen in figure 2(b). A clear 1332 $\mathrm{cm}^{-1}$ peak is seen with some evidence of a $1450 \mathrm{~cm}^{-1}$ band due to $\mathrm{sp}^{2}$ carbon.

The conductivity of NCD films can be controlled by the addition of TMB into the gas phase. NCD is intrinsically very resistive, being fundamentally diamond with added leakage due to grain boundary conduction. Adding TMB to these films makes them semiconducting with activation energy $0.37 \mathrm{eV}$, at higher levels hopping conductions is observed with lowering activation energy until the metallic transition is reached ${ }^{5}$. These doping levels obviously have an adverse effect on visible transmission, with metallically doped NCD of conductivity over $100 \Omega^{-}$ ${ }^{1} \mathrm{~cm}^{-1}$ having a transmission of around $50 \%$. At lower doping levels, very transparent films can be deposited on a variety of glasses and quartz as shown on figure 3 .
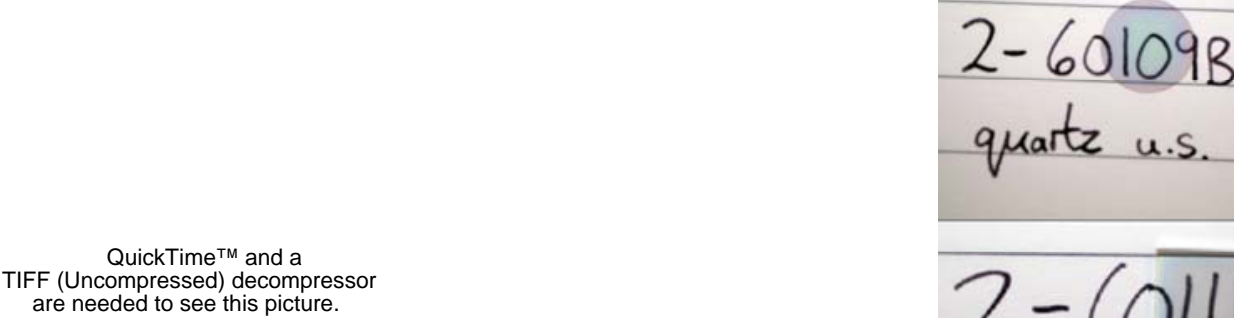

are needed to see decompressor
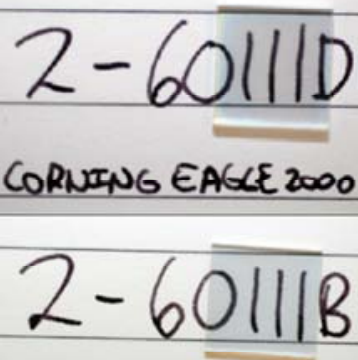

SCHOTT AF 45

Figure 3: Transmission of NCD films on various transparent substrates

The films shown in figure 3 show very high transparency even when moderately doped. This is due to the very thin nature of the film, less than $150 \mathrm{~nm}$. Thus NCD diamond films can be reasonably transparent and semiconducting. For transparent electrodes, the higher doping levels leads to reduced transparency as already mentioned, but for many biological applications, this transparency is still acceptable. The growth of NCD on some glasses such as Schott AF45 requires lower deposition temperatures, without the lower associated growth rates.

\section{POTENTIAL APPLICATIONS}

NCD films have many potential niche applications in biology. For the majority of biological applications, a very thin film of NCD is required. In order to grow these films the nucleation density must be very high, at least $>10^{11} \mathrm{~cm}^{-2}$. This allows very low surface roughness and no pinholes, which are disastrous for electrochemical applications. The first 
obvious application of these films is as electrochemical electrodes ${ }^{6}$, or more specifically optically transparent electrochemical electrodes ${ }^{7}$. For many applications, NCD diamond does not need to be doped. Biological windows of NCD coated quartz or glasses could be interesting for cell growth studies, as they exhibit many of the surface properties of single crystal diamond such as tunable wettabilty to the nanoscale ${ }^{8}$. These windows are ideal for cell proliferation experiments as a compliment to work done on single crystal diamond ${ }^{9}$. For Atomic Force Microscopy, the growth of diamond in sacrificial silicon molds can produce sharp tips with unrivalled hardness. These AFM tips can be doped with boron for electrochemical access and have significant advantages over conventional AFM tips due to their extreme chemical inertness, sharpness and wear resistance. The stability of functionalisation strategies on diamond surfaces is of advantage for all biological applications and is significantly better than any other semiconductor. For piezoelectric applications, diamond has many advantages. The aforementioned stability is of crucial importance, as tethered molecules that become unstable and removed from the surface could result in false negative signals in pathogen or immunosensing. Surface Acoustic Wave devices are of interest due to the high velocity of the SAW wave on diamond. This results in a very sensitivity, but unfortunately the applications are limited to biosensing in air. For liquid applications, the simplest realizable structure is the quartz microbalance. This device can sense mass loading effects down to below $5 \mathrm{ng} / \mathrm{cm}^{-2}$. Unfortunately the curie point of quartz is well below diamond deposition temperatures, resulting in a non piezoelectric phase after the growth process. By replacing the quartz with a high temperature stable piezoelectric we have been able to demonstrate a prototype diamond coated thickness shear mode resonator, as a replacement for the quartz microbalance. These devices have significant advanatages over the conventional quartz microbalance devices with metal electodes. Diamond adds to these devices, the widest electrochemical window, the most stable surface for functionalisation, extreme chemical inertness and tunable wettabilty at the nanoscale.

\section{CONCLUSIONS}

Nanocrystalline diamond has many of the extreme properties of diamond which are very interesting for biological applications. For real applications, NCD still remains within the "niche" areas such as biological windows, Conductive AFM tips and piezoelectric biosensing.

\section{ACKNOWLEDGMENTS (OPTIONAL)}

This work was financially supported by the IWT-SBO-project No. 030219 "CVD Diamond", by the IAP-P5/01 project "Quantum Size Effects in Nanostructured Materials" and the EU FP6 Marie Curie RTN "DRIVE", MRTN-CT-2004-512224. KH is a Postdoctoral Fellow of the Research Foundation-Flanders (FWO-Vlaanderen). 


\section{REFERENCES}

$1 \quad$ M. A. Prelas, G. Popovici, and L. K. Bigelow, Handbook of Industrial Diamonds and Diamond Films. (Marcel Dekker Inc, New York, 1998), p.1214.

2 W. Yang, O. Auciello, J. E. Butler, Cai W., J. A. Carlisle, J. Gerbi, D. M. Gruen, T. Knickerbocker, T.L. Lasseter, J. N. Russell, L.M. Smith, and R. J. Hamers, Nature Materials 1 (4), 253-257 (2002).

3 O. A. Williams and R. B. Jackman, Semicond. Sci. Technol. 18 (3), S34-S40 (2003).

4 J. A. Garrido, A. Hardl, S. Kuch, M. Stutzmann, O. A. Williams, and R. B. Jackmann, Applied Physics Letters 86 (7), 73504 (2005).

5 M. Nesladek, D. Tromson, C. Mer, P. Bergonzo, P. Hubik, and J. J. Mares, Applied Physics Letters 88 (23), 232111 (2006).

6 B. Fausett, M. C. Granger, M. L. Hupert, J. Wang, G. M. Swain, and D. M. Gruen, Electroanalysis 12 (1), 7-15 (2000).

7 J. Stotter, J. Zak, Z. Behier, Y. Show, and G. M. Swain, Analytical Chemistry 74 (23), 5924-5930 (2002).

$8 \quad$ Y. Kaibara, K. Sugata, M. Tachiki, H. Umezawa, and H. Kawarada, Diamond and Related Materials 12 (3-7), 560-564 (2003); K. Sugata, M. Tachiki, T. Fukuda, H. Seo, and H. Kawarada, Jpn. J. Appl. Phys. Part 1 - Regul. Pap. Short Notes Rev. Pap. 41 (7B), 4983-4986 (2002).

9 Christian G. Specht, Oliver A. Williams, Richard B. Jackman, and Ralf Schoepfer, Biomaterials 25 (18), 4073-4078 (2004). 